"an affecting, sobering account of a life shaped by, yet transcending, a mental-health condition and treatment".

The portrayal of mental-health conditions (or, to be less semantically guarded, mental illnesses) in the media and popular culture has a significant influence on the way that many people view both the conditions and those who have them. Next to lawyers and police officers, physicians are among the most frequently fictionalized professionals, and psychiatrists feature heavily. Despite the well-quoted statistic that one in four people will experience a mental illness at some point in their lives, the medical reality is something more easily viewed at a distance, peeking through the silver screen at the misery of somebody else. When that picture is distorted, the result is fear and mistrust, not just of the mental-health professionals but also of their patients.

So why do Hollywood and the rest of popular culture often get psychiatric medicine so wrong? Why do we still talk of the psychiatrist's couch when most people who see a psychiatrist never sit on one?

Too many accounts show only the illness, or at least a crude stereotype of it, not the person. And, crucially, they do not show how the person can often improve with the right treatment. Miracle cures are as rare in mental as in physical medicine, but many (perhaps most) people with psychiatric problems who receive the right kind of help can start to feel better. It is important to acknowledge and portray this.

A study published in February in the journal Social Science \& Medicine (E. E McGinty et al. Soc. Sci. Med. http://doi.org/2v4; 2015), for example, suggested that stories about people who were successfully treated for depression, schizophrenia and drug addiction reduced negative attitudes towards mental illness in those who heard them. Tales of the same problems left untreated produced more willingness to discriminate against sufferers. This discrimination and prejudice is known to act as a barrier to people's recovery, because they can experience

social rejection when word of their condition gets around.

Perhaps here is a way to tackle the stigma of mental illness. It is not enough for scientists, journalists and campaigners to 'raise awareness' by highlighting the reality of such conditions, and by publicly criticizing those who misuse the terms and language of psychiatric medicine. It is not enough to point out that phrases such as 'schizophrenic foreign policy' and 'a little bit OCD' are offensive because they misrepresent

"The way to break the destructive cycle, to treat the $O C D$, is to resist the ritual." and trivialize genuine and serious suffering. It is not enough to emphasize the biological basis of mental illness. To truly change public attitudes, the message must go out more often that this suffering can be alleviated.

In doing so, a powerful cultural myth must be challenged: that mental illness is a gift and comes with benefits. From the supposed enhanced creativity and meaningful visions of those with schizophrenia to the claimed cognitive wizardry and insight of people with autism, mental-health conditions are too often presented as just another way of seeing the world. This sense of instant karma might soften the blow to audiences, to some patients even, but it feeds the damaging impression that psychiatrists are out to rob special people of their gifts and unique potential. It is hard enough for people with mental-health problems to seek help, without their fearing those who are best placed to provide it.

What is so funny about OCD? The answer, Cefalu concluded, is the incongruity of the condition: the harder a sufferer tries to help themselves with comforting rituals, the worse their torment becomes. The way to break the destructive cycle, to treat the OCD, is to resist the ritual, to stop playing to the crowd. That was what Laura Jane Dean managed to do. That was her treatment. That is her story. And stories do not have to be funny to have a happy ending.

\section{Strike a chord}

\section{The latest episode of the Nature Audiofile podcast looks at how music inspires science.}

\section{“M} athematics and music! The most glaring possible opposites of human thought! And yet connected, mutually sustained!" Thus enthused German scientist Hermann von Helmholtz in an 1857 essay on harmony in music.

Today, mathematicians, ecologists and physicists search for harmony alongside musicians, if not always explicitly. But the link used to be even stronger.

A typical academic curriculum circa 1600 - and for centuries before - blended music and (what we would call) science to a degree rarely seen in today's undergraduate syllabi. The four subjects of the 'quadrivium' were arithmetic, geometry, astronomy and music.

Today's scientific training programmes tend to leave music out. They have changed in other ways too, of course, and for the better. Although some universities offer degrees in mathematics and music, or physics and music, modern scientists more often miss the chance to seek musical connections.

"Many people think music is a charming accompaniment to thought," says musician and scientist Peter Pesic of St John's College in Santa Fe, New Mexico, in the latest episode of Nature's sound-science podcast, Audiofile (go.nature.com/xhluk3). "But developments in music," he notes, "influence other aspects of human thought."

The podcast - one of a series - contains plenty of musical stories from the history of science. Take the astronomer Johannes Kepler, who was preoccupied with the motions of the planets. He was desperate to find harmony that he felt sure existed in the way the Universe was set up. In 1619, he produced a giant volume called Harmonices Mundi, or The Harmony of the World. In one particularly musical moment, he expresses planetary motions in musical notation - the orbits of Mercury, Venus and their neighbours spun out into crotchets and quavers. You can hear the resulting song on the podcast. Without such explicitly musical thinking, it is possible that Kepler may never have arrived at his third law of planetary motion - the relationship between a planet's distance from the Sun and the time it takes to orbit.

Then there is the tantalizing suggestion that Galileo Galilei's musical father might have influenced the way his son thought about science. In the late 1580s, Vincenzo Galilei carried out an experiment on the sounds made by strings held at different tensions. Vincenzo's home-made experiments could well have instilled in his son the idea of looking at a physical system to produce a hypothesis, rather than retrofitting the one to the other.

Musical analogies continue to help scientists to make sense of tricky concepts. String theorists speak of tiny vibrating strings instead of point-like particles. The comparison with stringed instruments is easy to see; the strings represent elementary particles in the same way that the strings on a guitar make different notes.

Musical inspiration can often remain buried under the surface of scientific work; there might not seem to be an immediate harmony between, say, a genome-wide association study and one of Erik Satie's études. But if music remains apart from the sciences, there is much we might lose. Anecdotally, neuroscientists talk of musical theory aiding the analysis of brain patterns. And a theory of strings is more intelligible to some than a theory of tiny, massless, vibrating subatomic squiggles.

Looking too hard for harmony might be misleading if the real picture turns out to be more discordant. But, as explained in the podcast by Jim Bennett, emeritus director of the Museum of the History of Science in $\rightarrow$ NATURE.COM To comment online, click on Editorials at: go.nature.com/xhunqv
Oxford, UK, music has already provided a great template for interpreting our surroundings: "The insight, which plausibly came from music, that the world has a mathematical blueprint is fundamental to science." - 